Published in final edited form as:

Med Chem. 2012 May ; 8(3): 392-400.

\title{
Four-Component Synthesis of 1,2-Dihydropyridine Derivatives and their Evaluation as Anticancer Agents
}

\author{
Mohamed A. O. Abdelf-Fattah ${ }^{1}$, Mahmoud A. M. El-Naggar ${ }^{1}$, Rasha M. H. Rashied ${ }^{1}$, Bernard \\ D. Gary ${ }^{2}$, Gary A. Piazza ${ }^{2}$, and Ashraf H. Abadi ${ }^{1, *}$ \\ ${ }^{1}$ Department of Pharmaceutical Chemistry, Faculty of Pharmacy and Biotechnology, German \\ University in Cairo, Cairo 11835, Egypt \\ ${ }^{2}$ Mitchell Cancer Institute, University of South Alabama, 1660 Springhill Avenue, Suite 3029, \\ Mobile AL 36604-1405
}

\section{Abstract}

Two series of compounds with the general formula of 4,6-diaryl-2-oxo-1,2 dihydropyridine-3carbonitriles and their isosteric imino derivatives were synthesized through a one pot reaction of acetophenone, aldehyde and ammonium acetate with ethyl cyanoacetate or malononitrile, respectively. The synthesized compounds were evaluated for tumor cell growth inhibitory using the human HT-29 colon and MDA-MB-231 breast tumor cell lines. Compound 4-(2Ethoxyphenyl)-2-imino-6-(4-fluorophenyl)-1,2-dihydropyridine-3 carbonitrile (6) showed IC $_{50}$ value of $0.70 \mu \mathrm{M}$ versus HT-29. Meanwhile, compound 4-(2-Hydroxyphenyl)-2-imino-6-(4fluorophenyl)-1,2-dihydropyridine-3-carbonitrile (4) showed $\mathrm{IC}_{50}$ value of $4.6 \mu \mathrm{M}$ versus MDAMB-231. Docking compound 10 to possible molecular targets, survivin and PIM1 kinase showed appreciable interactions with both, which suggest possible targets for the antitumor activity of this novel class of anticancer compounds.

\section{Keywords}

Multicomponent reactions; 1,2-dihydropyridine; cancer

\section{INTRODUCTION}

In times where a premium is put on speed, diversity, and efficiency in the drug discovery process, MCRs strategies offer significant advantages over conventional linear-type syntheses The idea of MCRs is to create a multiplicity of compounds and then testing their biological activity on a range of molecular targets, so that large number of molecules are available to identify the most promising lead compounds for preclinical development. Not to mention also the new world-wide movement of adopting the philosophy of green chemistry that emphasizes the importance of saving initial reactants, costs and waste. Thus MCR is one of the ideal choices by chemists for drug discovery [1-4].

\footnotetext{
*Address correspondence to these authors at the Department of Pharmaceutical Chemistry, Faculty of Pharmacy and Biotechnology, German University in Cairo, Cairo 11835, Egypt; Tel: 202-27090716; Fax: 202-27581041; ashraf.abadi@ guc.edu.eg.
} 
Compounds within the 3-cyano-4,6-diaryl-2(1H)imino or oxopyridines series represent a good example for those derivatives that can be developed utilizing MCR approaches. There is recently a great interest in this series of compounds due to their diverse pharmacological activities, such as antimicrobials against Gram positive and Gram negative microorganisms [5], antidepressant [6], cardiotonic [7], and anticancer [8,9] activities.

The tumor cell regulatory proteinsPIM1 kinase and survivin may serve as targets for the anticancer activity of this class of compounds.

PIM1 kinase is an oncogenic protein belonging to a family of proteins containing the homologues PIM-2 and PIM-3. PIM1 kinase is transcriptionally regulated by mitogens, cytokines, and various growth factors. PIM1 kinase is involved in the phosphorylation and activation of a large number of endogenous substrates that are associated with cell division and proliferation $[10,11]$.

Survivin is a member of a family of proteins known as Inhibitors of Apoptosis (IAP) and recent studies have shown that survivin is over expressed in many common human cancers and may be an important mediator of sensitivity to chemotherapy. Moreover, survivin has a very significant role in mitotic cell division where its expression pattern is cell cycledependent. In addition, survivin is one of the chromosomal passenger proteins; it complexes and localizes to Aurora B kinase in the mitotic machinery [12-14].

Different candidates belonging to 3-cyano-4,6-diaryl-2(1H) oxopyridines series have been recently shown promising anticancer activity through interfering with PIM1 kinase and surviving [11,12].

To explore the scope of this class of compounds, herein, we are reporting about thirty new derivatives with the general formula of 4,6-diaryl-2-imino (oxo)-1,2 dihydropyridine-3carbonitriles and their evaluation for anticancer activity, structure activity relationships, and interaction with potential molecular targets.

\section{RESULTS AND DISCUSSION}

\section{Chemistry}

The general synthesis of the 4,6-diaryl-2-oxo-1,2-dihydropyridine-3-carbonitrile and their 2imino counterparts is illustrated in Scheme 1, whereby the final products were obtained utilizing a multi-component reaction technique, a subtype of combinatorial reaction approach through refluxing the respective aldehyde with the corresponding ketone, ammonium acetate and ethyl cyanoacetate in ethanol for 16 to 18 hours, to afford the formation of the 2-pyridone derivative, or malononitrile to afford the iminopyridine derivative. All compounds were analyzed using ${ }^{1} \mathrm{H}-\mathrm{NMR}$, FT-IR spectroscopy, mass spectroscopy and elemental analysis. For mass spectroscopy, it was observed that in the majority of the compounds, the base peak was the molecular ion peak, indicating their stability.

Infrared spectra for the iminopyridine derivatives showed bands at the range of $3100-3400$ $\mathrm{cm}^{-1}$ corresponding to the $\mathrm{NH}$ stretching. A very characteristic band appeared at the range 
of $2100-2250 \mathrm{~cm}^{-1}$ corresponding to the cyano stretching. The 2-pyridone derivatives showed an extra band at the range of $1640-1710 \mathrm{~cm}^{-1}$ for the carbonyl stretching.

As for the ${ }^{1} \mathrm{H}$-NMR spectra, the diaryl substitution of the synthesized compounds led to the appearance of aromatic protons as multiplet peaks at chemical shifts $(\delta) 7.00-8.40 \mathrm{ppm}$. A singlet peak at around 6.50-7.00 ppm is characteristic for the aromatic proton at position 5 of the pyridone or iminopyridine ring. Compounds bearing methoxy substituent showed a characteristic singlet appeared at about $3.90 \mathrm{ppm}$, while those with the ethoxy substituent showed a characteristic triplet signal appeared at $1.25-1.30 \mathrm{ppm}$, and a quartet appeared at 4.03-4.13 ppm. Distances between peaks were described by coupling constants $(J)$.

\section{Biology}

All synthesized compounds were tested for their in vitro tumor cell growth inhibitory activity using the human HT-29 colon cancer cell line and breast cancer cell line MDAMB-231. Most of compounds were evaluated in 2 steps; first, the percentage inhibition at a screening dose of $50 \mu \mathrm{M}$ was performed in triplicate, then for compounds displaying a percentage of inhibition $>50 \%$ were evaluated by testing a range of 10 concentrations with at least two replicates per concentration to calculate an $\mathrm{IC}_{50}$ value. The results are summarized in Table 1.

In this study the effect of three major structure features on the anticancer activity of the synthesized candidates could be studied, namely the effect of the imino group on position 2 of the pyridine ring versus its 2-oxo isostere, the effect of the position of the fluorine atom on the phenyl ring at position 6 of the pyridine, and finally the nature of the substituent on the phenyl ring at position 4 of the pyridine ring.

Starting with the anticancer activity using the human HT-29 colon tumor cell line, 17 out of 30 synthesized candidates have showed appreciable tumor cell growth inhibitory activity. Generally, the presence of the imino group at position 2 of the pyridine ring gave higher potencies compared to the carbonyl isostere at the same position. This is clear when comparing the $\mathrm{IC}_{50} \mathrm{~s}$ of the imino derivatives to those of their 2-oxo analogues. The imino group may then be concluded as an important feature for the activity as it may interact with the target protein as both hydrogen bond donor and/ or acceptor.

In our synthesized candidates, the aryl group at position 4 of the pyridine ring carried either an oxygenated or halogenated substituent. As for the oxygenated substituents, they varied between 2-furanyl, 2-hydroxy phenyl, 2-methoxy phenyl, and 2-ethoxy phenyl, while the only halogenated substituent was 3,4-dichlorophenyl. The selection of the nature of the substituent at this position was based on previously published results by our group showing that candidates having negative electrostatic potential at this area exhibited anticancer activity $[8,9]$. The 2 -imino pyridine derivatives having the 2-ethoxy phenyl substituent at position 4 of the pyridine ring $\mathbf{6 , 1 6}$, and 26 have showed the highest potency with $\mathrm{IC}_{50}$ s of $0.70,1.5,2.5 \mu \mathrm{M}$ respectively, followed by those having 2-methoxy phenyl or 2-furanyl substituent at the same position $\mathbf{8 , 2 , 2 8 , 1 4}$, and 24 with $\mathrm{IC}_{50} \mathrm{~s}$ of $3.46,6.3,8.82,9.3,10.5$ $\mu \mathrm{M}$ respectively, and finally came the compounds having the 2-hydroxy phenyl substituent 4,12 , and 22 as the least potent with $\mathrm{IC}_{50} \mathrm{~s}$ of $12.7,>50,>50 \mu \mathrm{M}$ respectively. This may 
suggest that although the presence of certain degree of negative potential at this area is an important determinant for activity, the bulkiness of the substituent at this position is also important. It may be concluded that the bulky ethoxy function may be involved in additional hydrophobic interaction with the target protein and highly affects the degree of non coplanarity between the phenyl at position 4 and the pyridine ring that also seems to be a crucial factor for activity. It also decreases the free rotation degree of the phenyl ring, which can increase affinity and selectivity towards the target protein.

As for the candidates with a halogenated nature at position 4 of the pyridine ring, the 2imino derivatives with 3,4-dichloro phenyl at this position, namely 10, 20, and 30 displayed tumor cell growth inhibitory activity with $\mathrm{IC}_{50} \mathrm{~s}$ of $2.18,3.96$, and $5.74 \mu \mathrm{M}$ respectively. This confirms the necessity of having a certain degree of negative electrostatic potential at this area.

Regarding the position of the fluorine atom on the phenyl ring at position 6 of the pyridine ring, it was shown that the anticancer activity resides mainly in the candidates having the para or the meta fluoro substituent, while those with the ortho fluorine atom came as less active analogues. This is clear when comparing the potencies of compounds $\mathbf{6}, \mathbf{1 5}$ against $\mathbf{2 5}$, $\mathrm{IC}_{50} \mathrm{~s} 0.70,10.2,12.3 \mu \mathrm{M}$ respectively, compounds $\mathbf{1 8}, \mathbf{8}$ against $\mathbf{2 6}, \mathrm{IC}_{50} \mathrm{~s} 1.26,3.46,8.82$ $\mu \mathrm{M}$ respectively, and finally compounds $\mathbf{1 0}, 20$ against $\mathbf{3 0}, \mathrm{IC}_{50} \mathrm{~s} 2.18,3.96,5.74 \mu \mathrm{M}$ respectively. Actually, the majority of the synthesized compounds with the fluorine atom on the phenyl group at position 6 of the pyridine nucleus have generally shown to be more potent than other previously published analogues with bromine or chlorine atom in the same position, chart 2 . This may show the importance of highly electronegative atom such as the fluorine one in this area also. The small size of the fluorine atom relative to the chlorine or the bromine ones may also affect the fitting of the synthesized candidate into its binding pocket on the target protein.

Moving to the MDA-MB-231 breast cancer cell line, the active candidates did not show certain trends when varying the substituents on position 4 of the pyridine ring nor when varying the positions of the fluorine atom on the phenyl ring at position 6 . The highest potency was for compound 4 , having a 2-hydroxy phenyl at position 4 and para fluoro phenyl at position 6 of the pyridine ring. This compound came as one of the least active candidates on HT-29 colon cancer cell line and this may support the idea of having qualitative or quantitative differences of the different molecular targets in both cell lines.

Among the other possible targets for the synthesized candidates are PIM1 kinase and survivin. Consequently, docking experiments for one of the most active candidate $\mathbf{1 0}$ was performed to the active site of these proteins using MOE software [15].

The crystal structure of survivin (PDB ID code $1 \mathrm{~F} 3 \mathrm{H}$ ) showed homodimeric chains and with no ligand. As such, we applied global docking and the automatic site finder in the software. The two techniques came almost to the same types of interaction; namely indirect $\mathrm{H}$ bonding of the $\mathrm{NH}$ and $\mathrm{CN}$ through the solvent contacts with Arg18 and Gln92, Fig. (1). 
Docking compound 10 to the active site of PIM-1 (PDB ID code 2OBJ) reveals bidentate hydrogen bonding with the residue Lys67 and indirect $\mathrm{H}$ bonding through $\mathrm{H}_{2} \mathrm{O}$ molecules with Glu89 and Asp186, Fig. (2).

These intensive interactions with survivin and PIM1 kinase indicates that one or both targets might be responsible for the anticancer activity of this series of compounds. Such cross reactivity to structurally similar to compounds as previously reported [11, 12].

\section{EXPERIMENTAL}

\section{Chemistry}

All reactions were performed with commercially available reagents and used without further purification. All reactions were monitored by thin-layer chromatography (TLC) carried out on pre-coated silica gel plates and detection of the components was made by short and long UV light. Melting points were determined in open capillaries using a Buchi Melting Point B-540 apparatus and are uncorrected.1H-NMR spectra were recorded on Varian Mercury spectrometer at $300 \mathrm{MHz}$ using tetramethylsilane (TMS) as internal reference.

Chemical shift values are given in ppm at room temperature using DMSO-d6 and Chloroform as solvents; chemical shifts $(\delta)$ were reported in parts per million (ppm) downfield from TMS; multiplicities are abbreviated as: s: singlet; d: doublet; q: quartet; m: multiplet; dd: doublet of doublet; brs: broad singlet. Yields are not optimized. Elemental analyses were performed by the Microanalytical Unit, Faculty of Science, Cairo University; found values were within $\pm 0.4 \%$ of the theoretical ones, unless otherwise indicated.

\subsubsection{General Procedure for the Preparation of 4,6-diaryl-2-oxo-1,2} dihydropyridine-3-carbonitriles-The respective aromatic ketone $(1 \mathrm{mmol})$, together with the respective aromatic aldehyde $(1 \mathrm{mmol})$, ethyl cyanoacetate $(1 \mathrm{mmol})$, and ammonium acetate $(8 \mathrm{mmol})$ were dissolved in ethyl alcohol $(30 \mathrm{ml})$ and put under reflux for 10 to $12 \mathrm{~h}$. The precipitate obtained was filtered, washed with ethyl alcohol and dried. For the purification purpose, the precipitate was subjected to column chromatography on silica gel, eluting with wide range of eluting systems starting with methylene chloride to methylene chloride: methanol 9.85: 0.15 .

\section{6-(4-Flourophenyl)-4-(2-methoxyphenyl)-2-oxo-1,2-dihy-dropyridine-3- Carbonitrile} (1): Yield: $85 \%$; mp: $301-303{ }^{\circ} \mathrm{C}$; IR $\left(\mathrm{cm}^{-1}\right)$ : 3320, 2210, 1642; ${ }^{1} \mathrm{H}-\mathrm{NMR}\left(\mathrm{CDCl}_{3}\right): 3.91$ (s, $\left.3 \mathrm{H},-\mathrm{OCH}_{3}\right), 6.72$ (s, 1H, C5-pyridone), $7.06-7.78(\mathrm{~m}, 9 \mathrm{H}$, aromatic \& $\mathrm{NH})$; MS (EI): $\mathrm{m} / \mathrm{z} 320\left(\mathrm{M}^{+}, 100 \%\right)$; Anal. $\left(\mathrm{C}_{19} \mathrm{H}_{13} \mathrm{FN}_{2} \mathrm{O}_{2}\right) \mathrm{C}, \mathrm{H}, \mathrm{N}$.

\section{6-(4-Flourophenyl)-4-(2-hydroxyphenyl)-2-oxo-1,2-dihy-dropyridine-3-Carbonitrile} (3): Yield: $85 \%$; mp: $319-321^{\circ} \mathrm{C}$; IR $\left(\mathrm{cm}^{-1}\right): 3494,3339,2100,1705 ;{ }^{1} \mathrm{H}-\mathrm{NMR}$ (DMSO$\mathrm{d}_{6}$ ): 6.50 (s, 1H, C5-pyridone), 7.37-7.73 (m, 8H, aromatic); MS (EI): m/z 307 (M+1 100\%); Anal. $\left(\mathrm{C}_{18} \mathrm{H}_{11} \mathrm{FN}_{2} \mathrm{O}_{2}\right) \mathrm{C}, \mathrm{H}, \mathrm{N}$.

\section{4-(2-Ethoxyphenyl)-6-(4-flourophenyl)-2-oxo-1, 2-dihy-dropyridine-3-}

Carbonitrile(5): Yield: 70\%; mp: 261-263 ${ }^{\circ} \mathrm{C}$; IR $\left(\mathrm{cm}^{-1}\right)$ : 3230, 2223, 1638; ${ }^{1} \mathrm{H}-\mathrm{NMR}$ 
$\left(\right.$ DMSO-d $\left._{6}\right): 1.29\left(\mathrm{t}, 3 \mathrm{H}, \mathrm{J}=6.9 \mathrm{~Hz},-\mathrm{CH}_{3}\right), 4.12\left(\mathrm{q}, 2 \mathrm{H}, \mathrm{J}=6.9 \mathrm{~Hz},-\mathrm{OCH}_{2},\right), 7.05-7.10$ (m, $1 \mathrm{H}$, aromatic), $7.17-7.24(\mathrm{~m}, 1 \mathrm{H}$, aromatic) $7.33-7.96(\mathrm{~m}, 7 \mathrm{H}$, aromatic $\& \mathrm{NH})$; MS (EI): $\mathrm{m} / \mathrm{z} 334\left(\mathrm{M}^{+}, 100 \%\right)$; Anal. $\left(\mathrm{C}_{20} \mathrm{H}_{15} \mathrm{FN}_{2} \mathrm{O}_{2}\right) \mathrm{C}, \mathrm{H}, \mathrm{N}$.

6-(4-Flourophenyl)-4-(furan-2-yl)-2-oxo-1,2-dihydro-pyridine-3-carbonitrile(7): Yield: 21\%; mp: $307-310{ }^{\circ} \mathrm{C}$; IR $\left(\mathrm{cm}^{-1}\right): 3150,2221,1657 ;{ }^{1} \mathrm{H}-\mathrm{NMR}$ (DMSO-d $\left.{ }_{6}\right): 6.85$ (S, $1 \mathrm{H}$, C5-pyridone), 7.05 (s, 1H, aromatic), 7.40-7.35 (m, 1H, aromatic), $7.42(\mathrm{~d}, 1 \mathrm{H}, \mathrm{J}=2.1 \mathrm{~Hz}$, aroamtic), 7.72-7.69 (m, 1H, aromatic), 7.98-7.90 (m, 2H, aromatic), 8.12-8.09 (m, $1 \mathrm{H}$, aromatic), 12.63 (s, $1 \mathrm{H}, \mathrm{NH})$; $\mathrm{MS}(\mathrm{EI}): \mathrm{m} / \mathrm{z} 280\left(\mathrm{M}^{+}, 100 \%\right)$; Anal. $\left(\mathrm{C}_{16} \mathrm{H}_{9} \mathrm{FN}_{2} \mathrm{O}_{2}\right) \mathrm{C}, \mathrm{H}, \mathrm{N}$.

4-(3,4-Dichlorophenyl)-6-(4-fluorophenyl)-2-oxo-1,2-di-hydropyridine-3carbonitrile(9): Yield: $36 \%$; $\mathrm{mp}: 328-330^{\circ} \mathrm{C}$; IR $\left(\mathrm{cm}^{-1}\right)$ : 3092, 2218, $1642 ;{ }^{1} \mathrm{H}-\mathrm{NMR}$ (DMSO-d ${ }_{6}$ ): 6.93 (s, 1H, C5-pyridone), 7.35-7.43 (m, 2H, aromatic), 7.74 (d, 1H, J=9Hz, aromatic), $7.86(\mathrm{~d}, 1 \mathrm{H}, \mathrm{J}=9 \mathrm{~Hz}$, aroamtic), 7.97-8.07 (m, 3H, aroamtci), $12.95(\mathrm{~s}, 1 \mathrm{H}, \mathrm{NH})$; MS (EI): m/z $362\left(\mathrm{M}^{+}+4\right), \mathrm{m} / \mathrm{z} 360\left(\mathrm{M}^{+}+2\right), \mathrm{m} / \mathrm{z} 358\left(\mathrm{M}^{+}, 100 \%\right)$; Anal. $\left(\mathrm{C}_{18} \mathrm{H}_{9} \mathrm{FN}_{2} \mathrm{OCl}_{2}\right)$ $\mathrm{C}, \mathrm{H}, \mathrm{N}$.

6-(3-Flourophenyl)-4-(2-hydroxyphenyl)-2-ox0-1,2-dihy-dropyridine-3Carbonitrile(11): Yield: $73 \%$; mp: $317-319^{\circ} \mathrm{C}$; IR $\left(\mathrm{cm}^{-1}\right)$ : 3425, 2133, 1710; ${ }^{1} \mathrm{H}-\mathrm{NMR}$ (DMSO-d ${ }_{6}$ ): 6.42 (s, 1H, C5-Pyridone), 7.39-7.47 (m, 4H, aromatic), $7.58-7.95(\mathrm{~m}, 5 \mathrm{H}$, aromatic \& NH); MS (EI): m/z 307( $\left.\mathrm{M}^{+}, 100 \%\right)$; Anal. $\left(\mathrm{C}_{18} \mathrm{H}_{11} \mathrm{FN}_{2} \mathrm{O}_{2}\right) \mathrm{C}, \mathrm{H}, \mathrm{N}$.

6-(3-Flourophenyl)-4-(2-methoxyphenyl)-2-oxo-1, 2-dihy-dropyridine-3-Carbonitrile (13): Yield: $83 \%$; mp: $301-303^{\circ} \mathrm{C}$; IR $\left(\mathrm{cm}^{-1}\right): 3360,2217,1645 ;{ }^{1} \mathrm{H}-\mathrm{NMR}\left(\mathrm{CDCl}_{3}\right): 3.91$ (s, $3 \mathrm{H},-\mathrm{OCH}_{3}$ ), 6.50 (s, $1 \mathrm{H}, \mathrm{C} 5$-pyridone), $7.06-7.13$ (m, 3H, aromatic), 7.27- 7.78 (m, $6 \mathrm{H}$, aromatic \& $\mathrm{NH})$; MS (EI): m/z $320\left(\mathrm{M}^{+}, 100 \%\right)$; Anal. $\left(\mathrm{C}_{19} \mathrm{H}_{13} \mathrm{FN}_{2} \mathrm{O}_{2}\right) \mathrm{C}, \mathrm{H}, \mathrm{N}$.

\section{4-(2-Ethoxyphenyl)-6-(3-flourophenyl)-2-oxo-1, 2-dihy-dropyridine-3-}

Carbonitrile(15): Yield: 87\%; mp: $290-292^{\circ} \mathrm{C}$; IR $\left(\mathrm{cm}^{-1}\right)$ : 3281, 2222, 1645; ${ }^{1} \mathrm{H}-\mathrm{NMR}$ (DMSO-d $)_{6}$ ): 1.42 (t, 3H, - $\mathrm{CH}_{3}, \mathrm{~J}=6.9 \mathrm{~Hz},-\mathrm{CH}_{3}$ ), 4.16 (q, $2 \mathrm{H},-\mathrm{OCH}_{2^{-}}, \mathrm{J}=6.9 \mathrm{~Hz},-\mathrm{CH}_{2}-\mathrm{CH}_{3}$ ), 6.95 ( $\mathrm{S}, 1 \mathrm{H}, \mathrm{C5}$-pyridone), 7.03-7.14 (m, 2H, aromatic), 7.23-7.37 (m, 2H, aromatic), 7.37 - 7.77 (m, 4H, aromatic); MS (EI): m/z 334 (M+ 50.19\%), m/z 121 (100\%); Anal.

$\left(\mathrm{C}_{20} \mathrm{H}_{15} \mathrm{FN}_{2} \mathrm{O}_{2}\right) \mathrm{C}, \mathrm{H}, \mathrm{N}$.

6-(3-Flourophenyl)-4-(furan-2-yl)-2-oxo-1,2-dihydro-pyridine-3-carbonitrile(17): Yield: 30\%; mp: 307-308 ${ }^{\circ} \mathrm{C}$; IR ( $\left.\mathrm{cm}^{-1}\right)$ : 3126, 2219, 1657; ${ }^{1} \mathrm{H}-\mathrm{NMR}$ (DMSO-d 6 ): 6.85 (S, 1H, C5-pyridone), 7.13 (s, 1H, aromatic), 7.41 (t. 1H, J=9Hz, aromatic), 7.64-7.54 (m, 1H, aromatic), 7.80-7.71 (m, 3H, aromatic), 8.1 (s, 1H. aromatic), 12.65 (s, 1H, NH); MS (EI): $\mathrm{m} / \mathrm{z} 280\left(\mathrm{M}^{+}, 100 \%\right)$; Anal. $\left(\mathrm{C}_{16} \mathrm{H}_{9} \mathrm{FN}_{2} \mathrm{O}_{2}\right) \mathrm{C}, \mathrm{H}, \mathrm{N}$.

4-(3,4-Dichlorophenyl)-6-(3-fluorophenyl)-2-oxo-1,2-di-hydropyridine-3carbonitrile(19): Yield: $34 \%$; mp: $331-334^{\circ} \mathrm{C}$; IR $\left(\mathrm{cm}^{-1}\right)$ : 3094, 2225, 1642; ${ }^{1} \mathrm{H}-\mathrm{NMR}$ (DMSO-d $\left.{ }_{6}\right): 7.05$ (s, 1H, C5-pyridone) 7.36- $7.45(\mathrm{~m}, 1 \mathrm{H}$, aroamtic), 7.54- $7.63(\mathrm{~m}, 1 \mathrm{H}$, aroamtic), $7.72-7.79(\mathrm{~m}, 1 \mathrm{H}$, aromatic), $7.82(\mathrm{~s}, 1 \mathrm{H}$, aromatic), $7.84(\mathrm{~d}, 1 \mathrm{H}, \mathrm{J}=2.7 \mathrm{~Hz}$, aromatic), $7.87(\mathrm{~d}, 1 \mathrm{H}, \mathrm{J}=2.7 \mathrm{~Hz}$, aromatic), $8.05(\mathrm{~s}, 1 \mathrm{H}$, aromatic), $12.92(\mathrm{~s}, 1 \mathrm{H}, \mathrm{NH}) ; \mathrm{MS}$ (EI): m/z $362\left(\mathrm{M}^{+}+4\right), \mathrm{m} / \mathrm{z} 360\left(\mathrm{M}^{+}+2\right), \mathrm{m} / \mathrm{z}\left(\mathrm{M}^{+}, 100 \%\right)$; Anal. $\left(\mathrm{C}_{18} \mathrm{H}_{9} \mathrm{FN}_{2} \mathrm{OCl}_{2}\right) \mathrm{C}, \mathrm{H}, \mathrm{N}$. 
6-(2-Flourophenyl)-4-(2-hydroxyphenyl)-2-oxo-1,2-dihy-dropyridine-3-

Carbonitrile(21): Yield: 82\%; mp: $312-314^{\circ} \mathrm{C}$; IR $\left(\mathrm{cm}^{-1}\right)$ : 3268, 2133, 1690; ${ }^{1} \mathrm{H}-\mathrm{NMR}$ (DMSO-d ${ }_{6}$ ): 6.80 (s, 1H, C5-Pyridone), 7.32-7.45 (m, 5H, aromatic), 7.60-7.77 (m, 4H, aromatic); $\mathrm{MS}$ (EI): m/z 307( $\left.\mathrm{M}^{+}, 100 \%\right)$; Anal. $\left(\mathrm{C}_{18} \mathrm{H}_{11} \mathrm{FN}_{2} \mathrm{O}_{2}\right) \mathrm{C}, \mathrm{H}, \mathrm{N}$.

6-(2-Flourophenyl)-4-(2-methoxyphenyl)-2-oxo-1,2-dihy-dropyridine-3-

Carbonitrile(23): Yield: $91 \%$; mp: $308-310^{\circ} \mathrm{C}$; IR $\left(\mathrm{cm}^{-1}\right)$ : 3414, 2223, 1646; ${ }^{1} \mathrm{H}-\mathrm{NMR}$ (DMSO- $\left.\mathrm{d}_{6}\right): 3.82\left(\mathrm{~s}, 3 \mathrm{H},-\mathrm{OCH}_{3}\right), 6.86(\mathrm{~s}, 1 \mathrm{H}$, pyridone), 7.07-7.12 (m, $1 \mathrm{H}$, aromatic), 7.19 $-7.22(\mathrm{~m}, 1 \mathrm{H}$, aromatic), $7.32-7.41(\mathrm{~m}, 3 \mathrm{H}$, aromatic), $7.48-7.72(\mathrm{~m}, 4 \mathrm{H}$, aromatic \& $\mathrm{NH}) ; \mathrm{MS}(\mathrm{EI}): \mathrm{m} / \mathrm{z} 320\left(\mathrm{M}^{+}, 100 \%\right)$; Anal. $\left(\mathrm{C}_{19} \mathrm{H}_{13} \mathrm{FN}_{2} \mathrm{O}_{2}\right) \mathrm{C}, \mathrm{H}, \mathrm{N}$.

4-(2-Ethoxyphenyl)-6-(2-flourophenyl)-2-oxo-1,2-dihy-dropyridine-3-

Carbonitrile(25): Yield: $91 \%$; mp: $291-293^{\circ} \mathrm{C}$; IR $\left(\mathrm{cm}^{-1}\right)$ : 3392, 2222, $1643 ;{ }^{1} \mathrm{H}-\mathrm{NMR}$ (DMSO-d 6 ): 1.29 (t, 3H, J=6.95 Hz, - $\mathrm{CH}_{3}$ ), 4.13 (q, 2H, J=6.95Hz, $\left.-\mathrm{OCH}_{2}\right), 6.52(\mathrm{~s}, 1 \mathrm{H}$, C5-pyridone), 7.05-7.72 (m, 9H, aromatic \& NH); MS (EI): m/z $334\left(\mathrm{M}^{+}, 92.31 \%\right), \mathrm{m} / \mathrm{z} 63$ (100\%); Anal. $\left(\mathrm{C}_{20} \mathrm{H}_{15} \mathrm{FN}_{2} \mathrm{O}_{2}\right) \mathrm{C}, \mathrm{H}, \mathrm{N}$.

6-(2-Flourophenyl)-4-(furan-2-yl)-2-oxo-1,2-dihydro-pyridine-3-carbonitrile(27): Yield: 36\%; mp: 304-307 ${ }^{\circ}$; IR $\left(\mathrm{cm}^{-1}\right)$ : 3270, 2219, 1642; ${ }^{1} \mathrm{H}-\mathrm{NMR}$ (DMSO-d 6 ): 6.84 (m, 1H, C5-pyridone), 6.92 (s, $1 \mathrm{H}$, aromatic), $7.38(\mathrm{~m}, 2 \mathrm{H}$, aromatic), $7.72-7.57(\mathrm{~m}, 3 \mathrm{H}$, aromatic), 8.1 (t, $1 \mathrm{H}, \mathrm{J}=1.8 \mathrm{~Hz}$, aromatic), $12.72(\mathrm{~s}, 1 \mathrm{H}, \mathrm{NH}) ; \mathrm{MS}(\mathrm{EI}): \mathrm{m} / \mathrm{z} 280\left(\mathrm{M}^{+}, 100 \%\right) ;$ Anal. $\left(\mathrm{C}_{16} \mathrm{H}_{9} \mathrm{FN}_{2} \mathrm{O}_{2}\right) \mathrm{C}, \mathrm{H}, \mathrm{N}$.

4-(3,4-Dichlorophenyl)-6-(2-fluorophenyl)-2-oxo-1,2-dihydropyridine-3carbonitrile(29): Yield: $31 \%$; mp: $283-285^{\circ} \mathrm{C}$; IR $\left(\mathrm{cm}^{-1}\right)$ : 3125, 2221, 1642 ; ${ }^{1} \mathrm{H}-\mathrm{NMR}$ (DMSO-d $\left.{ }_{6}\right): 6.74$ (s, 1H, C5-pyridone), 7.44-7.33 (m, 2H, aromatic), $7.78-7.57(\mathrm{~m}, 3 \mathrm{H}$, aromatic), $7.85(\mathrm{dd}, 1 \mathrm{H}, \mathrm{J}=3 \mathrm{~Hz}$, aromatic), $8.02(\mathrm{t}, 1 \mathrm{H}, \mathrm{J}=3 \mathrm{~Hz}$, aroamtic), $12.99(\mathrm{~s}, 1 \mathrm{H}, \mathrm{NH})$; MS (EI): m/z $360\left(\mathrm{M}^{+}+4\right), \mathrm{m} / \mathrm{z} 360\left(\mathrm{M}^{+}+2\right), \mathrm{m} / \mathrm{z} 358\left(\mathrm{M}^{+}, 100 \%\right)$; Anal. $\left(\mathrm{C}_{18} \mathrm{H}_{9} \mathrm{Cl}_{2} \mathrm{FN}_{2} \mathrm{O}\right) \mathrm{C}, \mathrm{H}, \mathrm{N}$.

\subsubsection{General Procedure for the Preparation of 4,6-diaryl-2-imino-1,2-}

dihydropyridine-3-carbonitrile-The respective aromatic ketoe $(1 \mathrm{mmol})$, together with the respective aromatic aldehyde $(1 \mathrm{mmol})$, malononitrile $(1 \mathrm{mmol})$, and ammonium acetate $(8 \mathrm{mmol})$ were dissolved in ethyl alcohol $(30 \mathrm{ml})$ and put under reflux for $16-18 \mathrm{hrs}$. The precipitate obtained was filtered, washed with ethyl alcohol and dried. For the purification purpose, the precipitate was subjected to column chromatography on silica gel, eluting with methylene chloride or methylene chloride: methanol 9.9: 0.1.

6-(4-Flourophenyl)-2-imino-4-(2-methoxyphenyl)-1, 2-dihydropyridine-3Carbonitrile(2): Yield: 56\%; mp: 263- $265{ }^{\circ} \mathrm{C}$; IR $\left(\mathrm{cm}^{-1}\right)$ : 3360, 2221; ${ }^{1} \mathrm{H}-\mathrm{NMR}$ (DMSO$\left.\mathrm{d}_{6}\right): 3.80\left(\mathrm{~s}, 3 \mathrm{H},-\mathrm{OCH}_{3}\right), 6.88$ (s, $1 \mathrm{H}, \mathrm{C} 5$-pyridine). 7.06-7.11 (m, 1H, aromatic), 7.17-7.50 (m, 9H, aromatic \& NH); MS (EI): m/z $319\left(\mathrm{M}^{+}, 15 \%\right)$. m/z 121 (100\%); Anal. $\left(\mathrm{C}_{19} \mathrm{H}_{14} \mathrm{FN}_{3} \mathrm{O}\right) \mathrm{C}, \mathrm{H}, \mathrm{N}$.

6-(4-Flourophenyl)-4-(2-hydroxyphenyl)-2-imino-1,2-dihydropyridine-3-Carbonitrile (4): Yield: 82\%; mp: $293-295{ }^{\circ} \mathrm{C}$; IR $\left(\mathrm{cm}^{-1}\right): 3497,3367,2217 ;{ }^{1} \mathrm{H}-\mathrm{NMR}$ (DMSO-d 6 ): 6.91 
(s, 1H, C5-pyridine), 7.15-7.47 (m, 3H, aromatic), 7.64-7.70 (m, 1H, aromatic), $8.01-8.50$ (m, $6 \mathrm{H}$, aromatic \& NHs); MS (EI): m/z 306( $\left.\mathrm{M}^{+}, 100 \%\right)$; Anal. $\left(\mathrm{C}_{18} \mathrm{H}_{12} \mathrm{FN}_{3} \mathrm{O}\right) \mathrm{C}, \mathrm{H}, \mathrm{N}$.

4-(2-Ethoxyphenyl)-6-(4-flourophenyl)-2-imino-1, 2-dihydropyridine-3-Carbonitrile (6): Yield: $85 \%$; mp: $285-287{ }^{\circ} \mathrm{C}$; IR $\left(\mathrm{cm}^{-1}\right): 3373,2215 ;{ }^{1} \mathrm{H}-\mathrm{NMR}\left(\mathrm{DMSO}_{6}\right): 1.27$ (t, $\left.3 \mathrm{H}, \mathrm{J}=6.9 \mathrm{~Hz},-\mathrm{CH}_{3}\right), 4.10$ (q, 2H, J=6.9 Hz, $-\mathrm{OCH}_{2}$ ), 6.86 (s, 1H, C5-pyridine), 7.04-7.49 (m, 10H, aromatic \& NHs); MS (EI): m/z 333(M+, 62.67\%). m/z 305 (100\%); Anal. $\left(\mathrm{C}_{20} \mathrm{H}_{16} \mathrm{FN}_{3} \mathrm{O}\right) \mathrm{C}, \mathrm{H}, \mathrm{N}$.

6-(4-Flourophenyl)-4-(furan-2-yl)-2-imino-1,2-dihydropyridine-3-carbonitrile(8): Yield: 31\%; mp: $158-159^{\circ} \mathrm{C}$; IR (cm ${ }^{-1}$ ): 3329, 2208; ${ }^{1} \mathrm{H}-\mathrm{NMR}$ (DMSO-d 6 ): 6.78 (s, 1H, C5pyridine), $6.98(\mathrm{~s}, 2 \mathrm{H}$, aromatic), $7.32-7.58(\mathrm{~m}, 4 \mathrm{H}$, aromatic), 8.05 (s, $1 \mathrm{H}$, aromatic), 8.15-8.22 (m, 2H, aromatic); MS (EI): m/z $279\left(\mathrm{M}^{+}, 100 \%\right)$; Anal. $\left(\mathrm{C}_{16} \mathrm{H}_{10} \mathrm{FN}_{3} \mathrm{O}\right) \mathrm{C}, \mathrm{H}, \mathrm{N}$.

4-(3,4-Dichlorophenyl)-6-(4-fluorophenyl)-2-imino-1,2-dihydropyridine-3carbonitrile(10): Yield: 35\%; mp: 197- $198{ }^{\circ} \mathrm{C}$; IR $\left(\mathrm{cm}^{-1}\right)$ : 3353, 2220; ${ }^{1} \mathrm{H}-\mathrm{NMR}$ (DMSOd6): 6.86 (s, 1H, C5-pyridine), 7.08 (s, 1H, aromatic), 8.05 (s, 1H, aromatic), 8.26-7.20 (m, $8 \mathrm{H}$, aromatic); MS (EI): m/z $362\left(\mathrm{M}^{+}+4\right), \mathrm{m} / \mathrm{z} 360\left(\mathrm{M}^{+}+2\right), \mathrm{m} / \mathrm{z} 358\left(\mathrm{M}^{+}, 100 \%\right)$; Anal. $\left(\mathrm{C}_{18} \mathrm{H}_{10} \mathrm{FN}{ }_{3} \mathrm{Cl}\right) \mathrm{C}, \mathrm{H}, \mathrm{N}$.

6-(3-Flourophenyl)-4-(2-hydroxyphenyl)-2-imino-1,2-dihydropyridine-3Carbonitrile(12): Yield:64\%; mp: $290-292{ }^{\circ} \mathrm{C}$; IR $\left(\mathrm{cm}^{-1}\right)$ : 3342, $2110 ;{ }^{1} \mathrm{H}-\mathrm{NMR}$ (DMSO$\left.\mathrm{d}_{6}\right)$ : 6.27 (s, $1 \mathrm{H}, \mathrm{C} 5$-pyridine), 7.27 (s, 2H, aromatic), $7.41-7.98(\mathrm{~m}, 8 \mathrm{H}$, aromatic \& NHs); MS (EI): $\mathrm{m} / \mathrm{z}$ 306( $\left.\mathrm{M}^{+}, 100 \%\right)$; Anal. $\left(\mathrm{C}_{18} \mathrm{H}_{12} \mathrm{FN}_{3} \mathrm{O}\right) \mathrm{C}, \mathrm{H}, \mathrm{N}$.

6-(3-Flourophenyl)-2-imino-4-(2-methoxyphenyl)-1, 2-dihydropyridine-3Carbonitrile(14): Yield: 40\%; mp: 291-293 ${ }^{\circ} \mathrm{C}$; IR $\left(\mathrm{cm}^{-1}\right)$ : 3349, $2221 ;{ }^{1} \mathrm{H}-\mathrm{NMR}$ (DMSO$\left.\mathrm{d}_{6}\right): 3.80\left(\mathrm{~s}, 3 \mathrm{H},-\mathrm{OCH}_{3}\right), 6.92(\mathrm{~s}, 1 \mathrm{H}$, pyridine), 7.06-7.11 (m, 1H, aromatic), 7.18-7.36 (m, $4 \mathrm{H}$, aromatic), 7.46-7.96 (m, 5H, aromatic \& NHs); MS (EI): m/z $319\left(\mathrm{M}^{+}, 100 \%\right)$; Anal. $\left(\mathrm{C}_{19} \mathrm{H}_{14} \mathrm{FN}_{3} \mathrm{O}\right) \mathrm{C}, \mathrm{H}, \mathrm{N}$.

4-(2-Ethoxyphenyl)-6-(3-flourophenyl)-2-imino-1, 2-dihydropyridine-3-Carbonitrile (16): Yield: 45\%; mp: $250-252{ }^{\circ} \mathrm{C}$; IR $\left(\mathrm{cm}^{-1}\right): 3359,2142 ;{ }^{1} \mathrm{H}-\mathrm{NMR}$ (DMSO-d $\left.{ }_{6}\right): 1.40$ (t, $3 \mathrm{H}, \mathrm{J}=6.97 \mathrm{~Hz},-\mathrm{CH}_{3}$ ), 4.15 (q, 2H, J=6.97Hz, -OCH2), 6.55 (s, 1H, C5-Pyridine), 7.03-7.81 (m, 10H, aromatic \& NHs); MS (EI): m/z 333( $\left.\mathrm{M}^{+}, 82.5 \%\right)$. m/z 304 (100\%); Anal. $\left(\mathrm{C}_{20} \mathrm{H}_{16} \mathrm{FN}_{3} \mathrm{O}\right) \mathrm{C}, \mathrm{H}, \mathrm{N}$.

6-(3-Flourophenyl)-4-(furan-2-yl)-2-imino-1,2-dihydropyridine-3carbonitrile(18): Yield: $28 \%$; mp: $248-250{ }^{\circ} \mathrm{C}$; IR $\left(\mathrm{cm}^{-1}\right)$ : 3357, 2207; ${ }^{1} \mathrm{H}-\mathrm{NMR}$ (DMSO$\mathrm{d}_{6}$ ): 6.78 (s, 1H, C5-pyridine), 7.01 (s, 2H, aromatic), 7.28-8.12 (m, 6H, aromatic),; MS (EI): m/z $279\left(\mathrm{M}^{+}, 100 \%\right)$; Anal. $\left(\mathrm{C}_{16} \mathrm{H}_{10} \mathrm{FN}_{3} \mathrm{O}\right) \mathrm{C}, \mathrm{H}, \mathrm{N}$.

4-(3,4-Dichlorophenyl)-6-(3-fluorophenyl)-2-imino-1,2-dihydropyridine-3carbonitrile(20): Yield: $22 \%$; $\mathrm{mp}: 215-216{ }^{\circ} \mathrm{C}$; IR $\left(\mathrm{cm}^{-1}\right)$ : 3355, 2216; ${ }^{1} \mathrm{H}-\mathrm{NMR}$ (DMSO$\left.\mathrm{d}_{6}\right): 6.89-6.96(\mathrm{~m}, 3 \mathrm{H}$, aromatic), $7.42-7.31(\mathrm{~m}, 1 \mathrm{H}$, aromatic), 7.46-8.05 (m, 6H, 
aromatic); MS (EI): m/z $361\left(\mathrm{M}^{+}+4\right), \mathrm{m} / \mathrm{z} 359\left(\mathrm{M}^{+}+2\right), \mathrm{m} / \mathrm{z} 357\left(\mathrm{M}^{+}, 100 \%\right)$; A-nal. $\left(\mathrm{C}_{18} \mathrm{H}_{10} \mathrm{FN}_{3} \mathrm{Cl}_{2}\right) \mathrm{C}, \mathrm{H}, \mathrm{N}$.

6-(2-Flourophenyl)-4-(2-hydroxyphenyl)-2-imino-1, 2-dihydropyridine-3-Carbonitrile (22): Yield: $77 \%$; mp: $278-280{ }^{\circ} \mathrm{C}$; IR $\left(\mathrm{cm}^{-1}\right)$ : 3420, 3331, 2213; ${ }^{1} \mathrm{H}-\mathrm{NMR}$ (DMSO-d 6 ): 6.8 (s, 1H, C5-Pyridine), 7.34-7.44 (m, 4H, aromatic), 7.52-7.59 (m, 1H, aromatic), 7.64-7.96 (m, 5H, aromatic \& NHs); MS (EI): m/z 306( $\left.\mathrm{M}^{+}, 100 \%\right)$; Anal. $\left(\mathrm{C}_{18} \mathrm{H}_{12} \mathrm{FN}_{3} \mathrm{O}\right) \mathrm{C}, \mathrm{H}, \mathrm{N}$.

6-(2-Flourophenyl)-2-imino-4-(2-methoxyphenyl)-1, 2-dihydropyridine-3-Carbonitrile (24): Yield: $71 \%$; mp: $289-291{ }^{\circ} \mathrm{C}$; IR $\left(\mathrm{cm}^{-1}\right)$ : 3301, 2100; ${ }^{1} \mathrm{H}-\mathrm{NMR}\left(\mathrm{DMSO}-\mathrm{d}_{6}\right): 3.80$ (s, $\left.3 \mathrm{H},-\mathrm{OCH}_{3}\right), 7.27-7.58(\mathrm{~m}, 7 \mathrm{H}$, aromatic), 7.76-7.79 (m, 1H, aromatic) 7.91-7.96 (m, 2H, aromatic \& NHs); MS (EI): m/z 319\%( $\left.\mathrm{M}^{+}, 25 \%\right) . \mathrm{m} / \mathrm{z} 227$ (100\%); Anal. $\left(\mathrm{C}_{19} \mathrm{H}_{14} \mathrm{FN}_{3} \mathrm{O}\right) \mathrm{C}$, $\mathrm{H}, \mathrm{N}$.

4-(2-Ethoxyphenyl)-6-(2-flourophenyl)-2-imino-1, 2-dihydropyridine-3-Carbonitrile (26): Yield: 64\%; mp: $248-250{ }^{\circ} \mathrm{C}$; IR $\left(\mathrm{cm}^{-1}\right)$ : 3330, 2113; ${ }^{1} \mathrm{H}-\mathrm{NMR}$ (DMSO-d 6 ): 1.29 (t, $3 \mathrm{H}, \mathrm{J}=6.9 \mathrm{~Hz},-\mathrm{CH} 3$ ), 4.11 (q, 2H, J = 6.9Hz, -OCH2,), 6.5 (s, 1H, C5-pyridine), 7.04-7.55 (m, $10 \mathrm{H}$, aromatic \& NHs); $\mathrm{MS}(\mathrm{EI}): \mathrm{m} / \mathrm{z} 333 \%\left(\mathrm{M}^{+}, 100 \%\right)$; Anal. $\left(\mathrm{C}_{20} \mathrm{H}_{16} \mathrm{FN}_{3} \mathrm{O}\right) \mathrm{C}, \mathrm{H}, \mathrm{N}$.

6-(2-Flourophenyl)-4-(furan-2-yl)-2-imino-1,2-dihydropyridine-3-carbonitrile (28): Yield: $27 \%$; mp: $105-107^{\circ} \mathrm{C}$; IR $\left(\mathrm{cm}^{-1}\right)$ : 3352, $2207 ;{ }^{1} \mathrm{H}-\mathrm{NMR}$ (DMSO-d $\mathrm{d}_{6}$ ): 6.60 (s, 1H, C5-pyridine), $7.11-6.64(\mathrm{~m}, 1 \mathrm{H}$, aromatic), 7.18-8.08 (m, 7H, aromatic); MS (EI):m/z $279\left(\mathrm{M}^{+}, 100 \%\right)$; Anal. $\left(\mathrm{C}_{16} \mathrm{H}_{10} \mathrm{FN}_{3} \mathrm{O}\right) \mathrm{C}, \mathrm{H}, \mathrm{N}$.

\section{4-(3,4-Dichlorophenyl)-6-(2-fluorophenyl)-2-imino-1,2-dihydropyridine-3-} carbonitrile(30): Yield: $21 \%$; mp: $107-109^{\circ} \mathrm{C}$; IR $\left(\mathrm{cm}^{-1}\right)$ : 3351,$2213 ;{ }^{1} \mathrm{H}-\mathrm{NMR}$ (DMSO$\mathrm{d}_{6}$ ): 6.98 (s, 1H, C5-pyridine), 7.20-7.83 (m, 7H, aromatic), 7.84-8.16 (m, 2H, aromatic); MS (EI):m/z $361\left(\mathrm{M}^{+}+4\right), \mathrm{m} / \mathrm{z} 359\left(\mathrm{M}^{+}+2\right), \mathrm{m} / \mathrm{z} 357\left(\mathrm{M}^{+}, 100 \%\right)$; Anal. $\left(\mathrm{C}_{18} \mathrm{H}_{10} \mathrm{Cl}_{2} \mathrm{FN}_{3}\right) \mathrm{C}$, $\mathrm{H}, \mathrm{N}$.

\section{BIOLOGY}

\section{Cell Cultures}

HT-29 tumor cells and MDA-MB-231 breast cancer cells were obtained from ATCC and were grown under standard cell culture conditions at $37{ }^{\circ} \mathrm{C}$ in a humidified atmosphere with 5\% CO2. Cells were grown in RPMI 1640 containing 5\% fetal bovine serum. Cell count and viability was determined by Trypan blue staining followed by hemocytometry. Only cultures displaying $>95 \%$ cell viability were used for experiments.

\section{Growth Assays}

Tissue culture treated microtiter 96-well plates were seeded at a density of 5000 cells/well. The plates were incubated for 18-24 h prior to any treatment. Cell viability was measured 72 $\mathrm{h}$ after treatment by the Cell Titer Glo Assay (Promega), which is a luminescent assay that is an indicator of live cells as a function of metabolic activity and ATP content. The assay was performed according to manufacturer's specifications. 


\section{MOLECULAR MODELING}

\section{Energy Minimization and Conformational Search Procedure}

The compounds were drawn on ChemSketch 11 and saved as mol file, the latter were subjected to energy minimization using Force Field MMFF94x by Molecular Operating Environment (MOE) software [15].

\section{Source of Target Proteins}

The Crystal structure of Human PIM-1 kinase complexed with its inhibitor reference compound (PDB ID code: 2OBJ), survivin (PDB ID code: 1F3H), were downloaded from the Protein data bank and opened with MOE software. The complexed inhibitor, if any, was removed when using the synthesized compounds as ligands for docking.

\section{Docking Procedure of PIM-1 Kinase}

The co-crystallized compound was selected, and the binding site was identified according to residues. Ligand interactions were computed for the X-ray co-crystallized compound to reveal the different types of interaction as a validation for the coming docking procedure. The co-crystallized compound was then removed and replaced by the synthesized compound. Default settings of MOE-Dock were used, including "Rotate Bonds" option in order to allow flexible ligand-rigid receptor docking. The scoring function was London $\mathrm{dG}$ with a replacement of Alpha Triangle. Thirty conformers of the ligand were retained with highest and best score by default. The top score ligand-receptor docking was then demonstrated by 2D ligand-receptor interactions.

\section{Docking Procedure of Survivin}

Survivin is an identical dimeric protein. The binding site was defined by selecting the residues Leu6, Lys15, Phe86, Val89, Phe93, Leu96, Leu98, Phe101, Leu102 and Leu104 as reported in Literature [12]. The docking procedure was done by the default settings of MOEDock.

\section{References}

1. Ganem B. Strategies for innovation in multicomponent reaction design. Acc Chem Res. 2009; 42:463-472. [PubMed: 19175315]

2. Zhang W. Fluorous-enhanced multicomponent reactions for making drug-like library scaffolds. Comb Chem High T Scr. 2007; 10:219-229.

3. Sheldon AR. Atom efficiency and catalysis in organic synthesis. Pure Appl Chem. 2000; 72:123346.

4. Liu L, Wei L, Lu Y, Zhang J. One-pot tandem catalysis: a concise route to fused bicyclic scaffolds from acyclic $\beta$-ketoesters and alkynyl aldehydes. Chemistry. 2010; 16:11813-7. [PubMed: 20809555]

5. Abdel-Aziz A, El-Subbagh HI, Kunieda T. Lewis acid-promoted transformation of 2alkoxypyridines into 2-aminopyridines and their antibacterial activity. Part 2: Remarkably facile CN bond formation. Bioorg Med Chem. 2005; 13:4929-35. [PubMed: 15993091]

6. Abdel-Latif N. Synthesis and antidepressant activity of some new coumarin derivatives. Sci Pharm. 2005; 73:195-216. 
7. Abadi AH, Al-Khamees HA. 3-Cyano-4,6-disubstituted-2(1H)-imino or oxopyridines: new antineoplastic agents with high selectivity towards leukemia cell lines. Arch, Pharm (Weinheim). 1998; 331:319-24. [PubMed: 9844579]

8. Abadi AH, Abouel-Ella DA, Lehmann J, Tinsley HN, Gary BD, Piazza GA, Abdel-Fattah MA. Discovery of colon tumor cell growth inhibitory agents through a combinatorial approach. Eur J Med Chem. 2010; 45:90-97. [PubMed: 19836860]

9. Abadi AH, Ibrahim TM, Abouzid K, Lehmann J, Tinsley HN, Gary BD, Piazza GA. Design synthesis and biological evaluation of novel pyridine derivatives as anticancer agents and phosphodiesterase 3 inhibitors. Bioorg Med Chem. 2009; 17:5974-5982. [PubMed: 19628397]

10. Pogacic V, Bullock AN, Fedorov O, Filippakopoulos P, Gasser C, Biondi A, Meyer-Monard S, Knapp S, Schwaller J. Structural analysis identifies imidazo [1,2-b]pyridazines as PIM kinase inhibitors with in vitro antileukemic activity. Cancer Res. 2007; 67:6916-6924. [PubMed: 17638903]

11. Cheney IW, Yan S, Appleby T, Walker H, Vo T, Yao N, Hamatake R, Hong Z, Wu JZ. Bioorg Med Chem Lett. 2007; 17:1679-1683. [PubMed: 17251021]

12. Wendt MD, Sun C, Kunzer A, Sauer D, Sarris K, Hoff E, Yu L, Nettesheim DG, Chen J, Jin S, Comess KM, Fan Y, Anderson SN, Isaac B, Olejniczak ET, Hajduk PJ, Rosenberg SH, Elmore SW. Identification and structure-activity relationships of substituted pyridones as inhibitors of Pim-1 kinase. Bioorg Med Chem Lett. 2007; 17:3122-3129. [PubMed: 17391963]

13. Zhonghong L, Lianjie L, Changqing Z, Ying H, Yu J, Yan L. The influence of survivin shRNA on the cell cycle and the invasion of SW480 cells of colorectal carcinoma. J Exp Clin Cancer Res. 2008; 27:20. [PubMed: 18637206]

14. Rankin WV, Henry CJ, Turnquist SE, Turk JR, Beissenherz ME, Tyler JW, Rucker EB, Knapp DW, Rodriguez CO, Green JA. Identification of survivin, an inhibitor of apoptosis, in canine urinary bladder transitional cell carcinoma. Vet Comp Oncol. 2008; 6:141-150. [PubMed: 19178674]

15. MOE, Chemical Computing Group Inc. http://www.chemcomp/com 


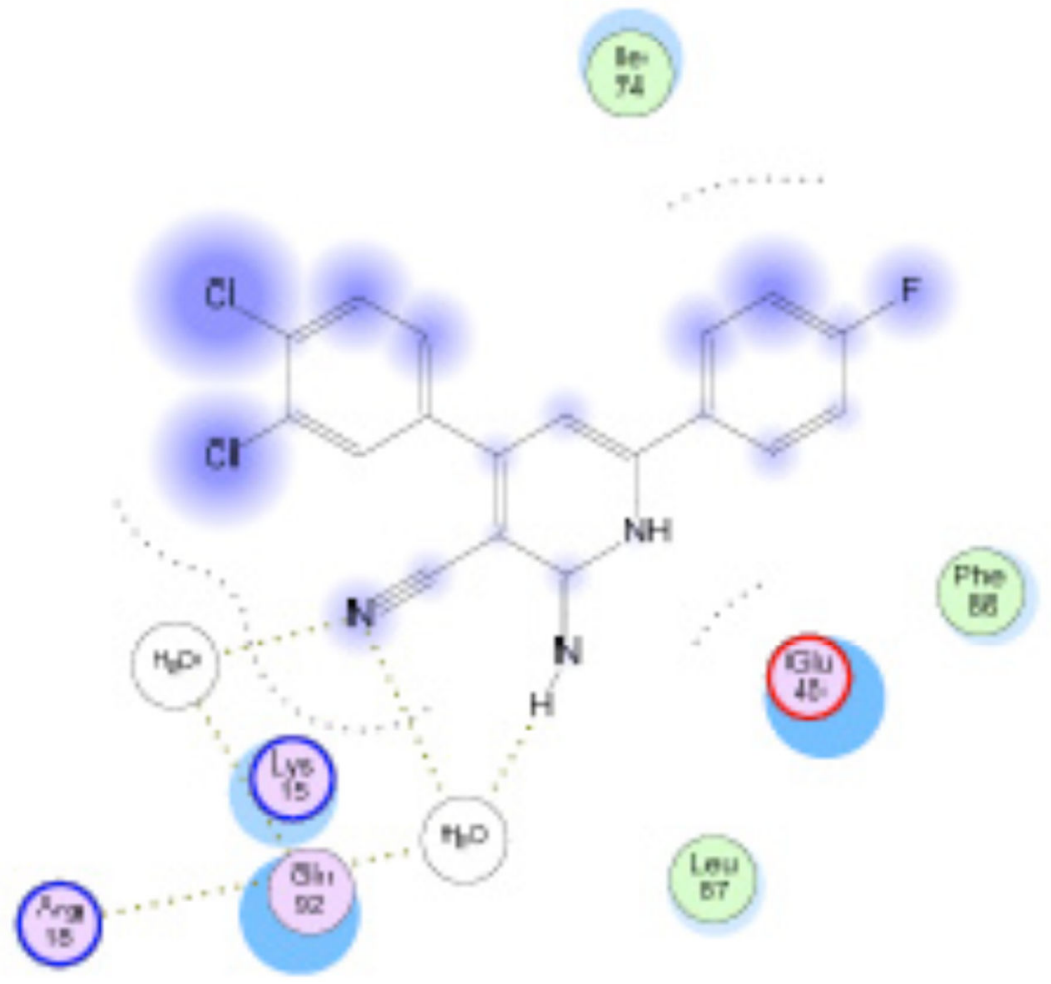

Fig. 1.

2D interaction of compound 10 with Survivin. 


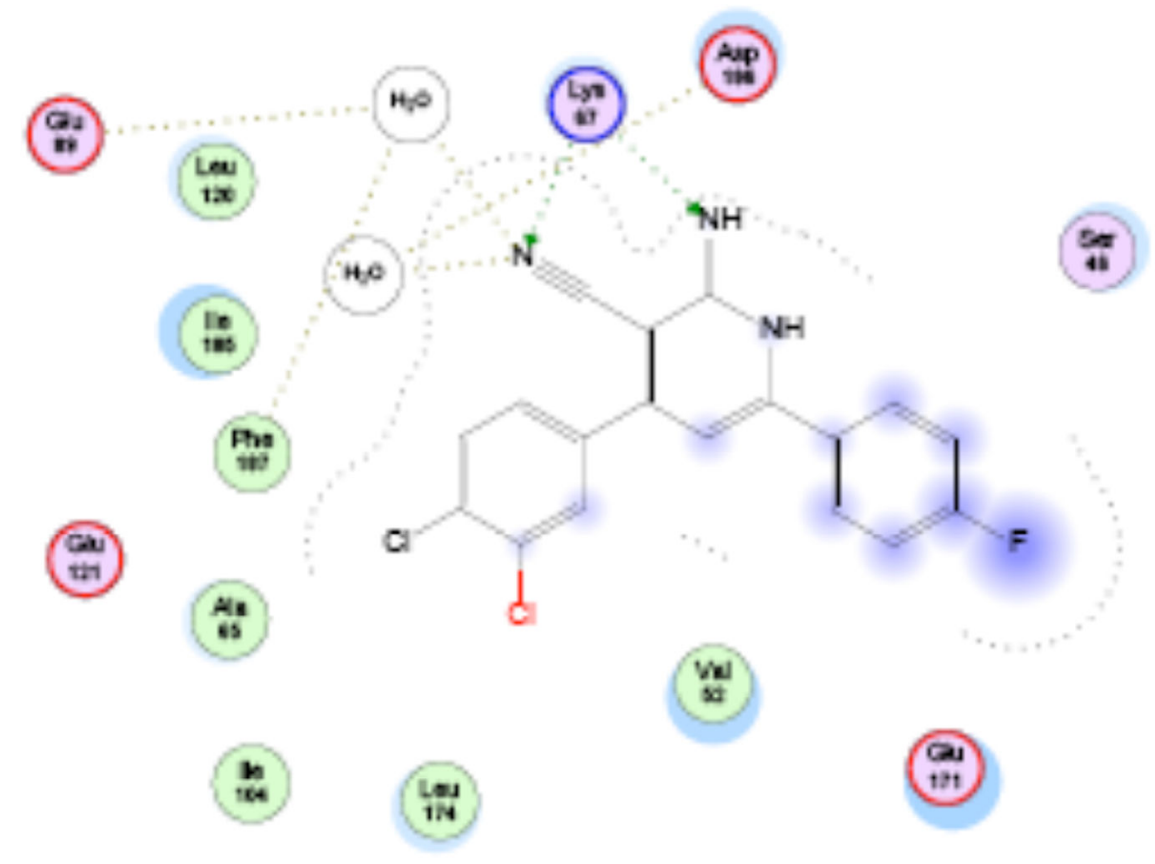

Fig. 2.

2D interaction of compound 10 with PIM1 Kinase. 


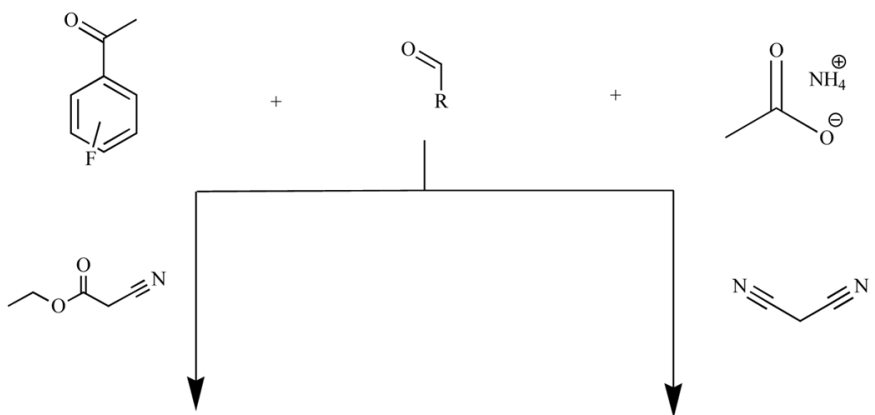<smiles></smiles><smiles></smiles>

$\mathrm{X}=\mathrm{O}$

$\mathrm{X}=\mathrm{NH}$

\begin{tabular}{|c|c|c|c|c|c|c|c|}
\hline Cpd & Fluoro Position & $\mathbf{R}$ & $\mathrm{x}$ & Cpd & $\begin{array}{l}\text { Fluoro } \\
\text { Position }\end{array}$ & $\mathbf{R}$ & $\mathrm{x}$ \\
\hline 1 & $4-\mathrm{F}$ & 2-methoxy phenyl & $\mathrm{o}$ & 16 & $3-\mathrm{F}$ & 2-ethoxy phenyl & $\mathrm{NH}$ \\
\hline 2 & $4-\mathrm{F}$ & 2-methoxy phenyl & $\mathrm{NH}$ & 17 & $3-\mathrm{F}$ & 2-furanyl & $\mathrm{o}$ \\
\hline 3 & $4-\mathrm{F}$ & 2-hydroxy phenyl & o & 18 & $3-\mathrm{F}$ & 2-furanyl & $\mathrm{NH}$ \\
\hline 4 & $4-\mathrm{F}$ & 2-hydroxy phenyl & $\mathrm{NH}$ & 19 & $3-\mathrm{F}$ & 3,4Dichlorophenyl & o \\
\hline 5 & $4-\mathrm{F}$ & 2-ethoxy phenyl & $\mathrm{O}$ & 20 & $3-\mathrm{F}$ & 3,4Dichlorophenyl & $\mathrm{NH}$ \\
\hline 6 & $4-\mathrm{F}$ & 2-ethoxy phenyl & $\mathrm{NH}$ & 21 & $2-\mathrm{F}$ & 2-hydroxy phenyl & $\mathrm{o}$ \\
\hline 7 & 4-F & 2-furanyl & o & 22 & 2-F & 2-hydroxy phenyl & $\mathrm{NH}$ \\
\hline 8 & $4-\mathrm{F}$ & 2-furanyl & $\mathrm{NH}$ & 23 & $2-\mathrm{F}$ & 2-methoxy phenyl & o \\
\hline 9 & $4-\mathrm{F}$ & 3,4Dichlorophenyl & $\mathrm{o}$ & 24 & $2-\mathrm{F}$ & 2-methoxy phenyl & $\mathrm{NH}$ \\
\hline 10 & $4-\mathrm{F}$ & 3,4Dichlorophenyl & $\mathrm{NH}$ & 25 & $2-\mathrm{F}$ & 2-ethoxy phenyl & $\mathrm{o}$ \\
\hline 11 & $3-\mathrm{F}$ & 2-hydroxy phenyl & o & 26 & $2-\mathrm{F}$ & 2-ethoxy phenyl & $\mathrm{NH}$ \\
\hline 12 & $3-\mathrm{F}$ & 2-hydroxy phenyl & $\mathrm{NH}$ & 27 & $2-\mathrm{F}$ & 2-furanyl & o \\
\hline 13 & $3-\mathrm{F}$ & 2-methoxy phenyl & o & 28 & $2-\mathrm{F}$ & 2-furanyl & $\mathrm{NH}$ \\
\hline 14 & $3-\mathrm{F}$ & 2-methoxy phenyl & $\mathrm{NH}$ & 29 & $2-\mathrm{F}$ & 3,4Dichlorophenyl & $\mathrm{o}$ \\
\hline 15 & $3-\mathrm{F}$ & 2-ethoxy phenyl & $\mathrm{o}$ & 30 & $2-\mathrm{F}$ & 3,4Dichlorophenyl & $\mathrm{NH}$ \\
\hline
\end{tabular}

\section{Scheme 1.}


<smiles>N#Cc1c(-c2cc(Cl)ccc2Cl)cc(-c2cc(Br)ccc2O)[nH]c1=O</smiles>

$\mathrm{IC}_{50}$ (Survivin): $0.8 \mu \mathrm{M}$<smiles>N#Cc1c(-c2ccccc2)cc(-c2cc(Br)ccc2O)[nH]c1=O</smiles>

$\mathrm{IC}_{50}(\mathrm{PIM} 1$ Kinase): $13 \mu \mathrm{M}$

Chart 1.

Some potential antitumor 3-cyano-4,6-diaryl-2(1H)imino or oxopyridines with their $\mathrm{IC}_{50} \mathrm{~s}$ against different possible molecular targets. 

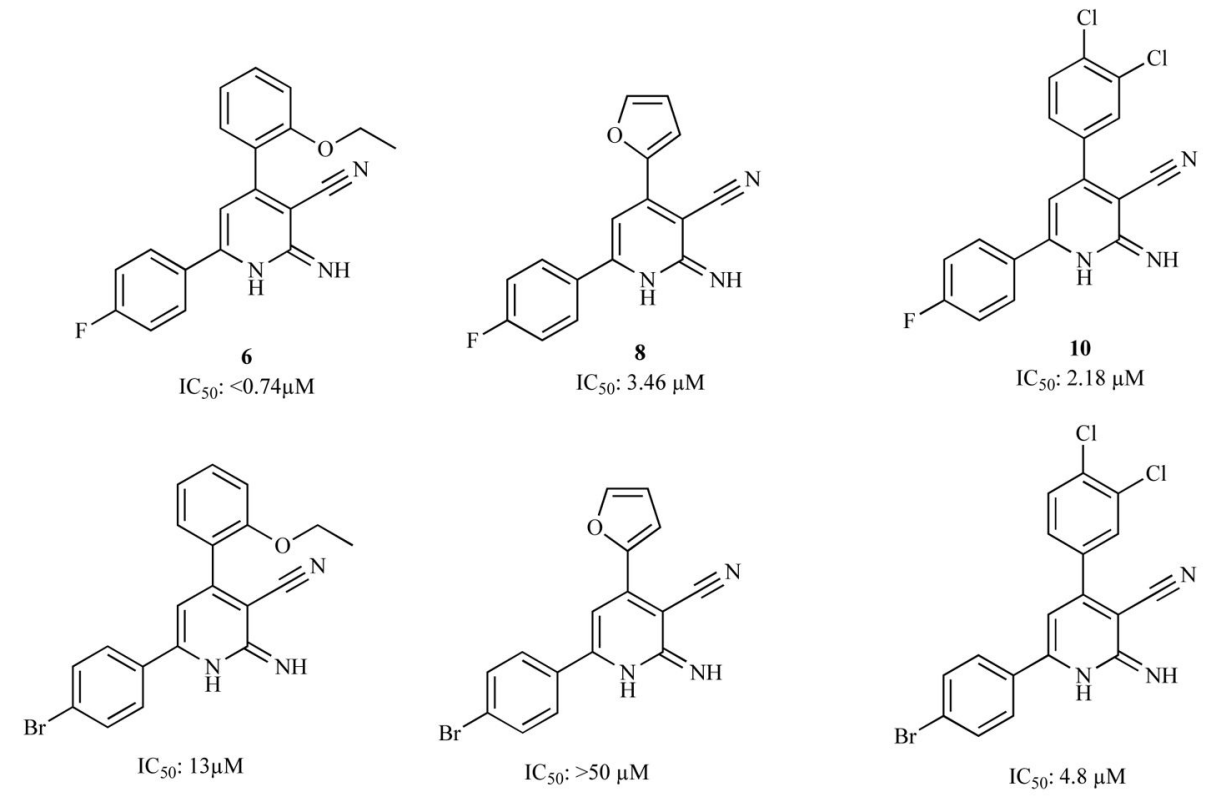

Chart 2.

Growth inhibitory potencies of some selected candidates relative to their brominated analogues versus HT-29 colorectal cancer cell lines [8,9]. 


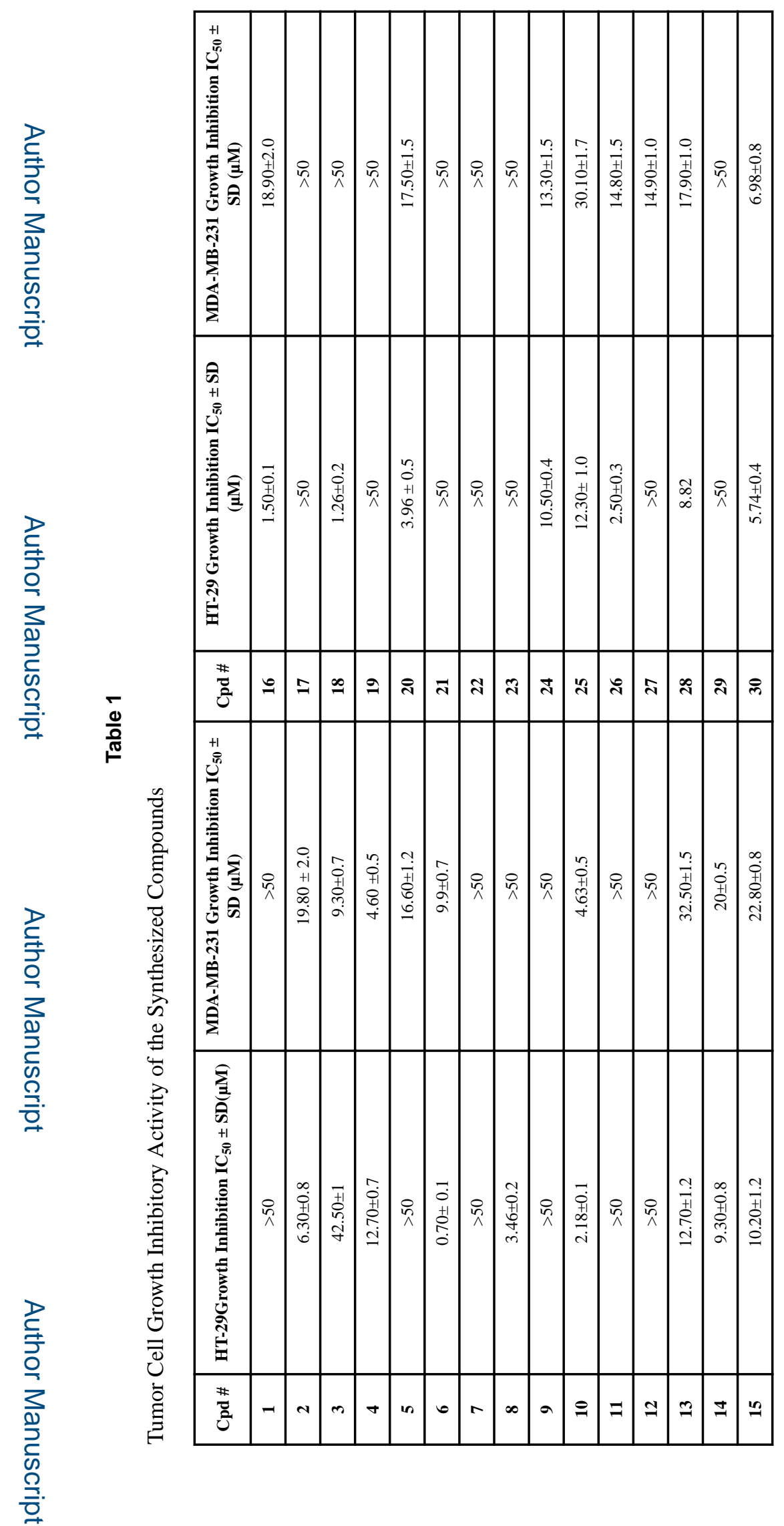

Med Chem. Author manuscript; available in PMC 2016 August 11. 